\title{
Healthcare, Sickness Absence, and Disability Pension Cost Trajectories in the First 5 Years After Diagnosis with Multiple Sclerosis: A Prospective Register-Based Cohort Study in Sweden
}

\author{
Korinna Karampampa ${ }^{1}$ (D) Hanna Gyllensten ${ }^{1,2} \cdot$ Fei Yang $^{1} \cdot$ Chantelle Murley $^{1} \cdot$ Emilie Friberg $^{1} \cdot$ Jan Hillert $^{1}$. \\ Kristina Alexanderson ${ }^{1}$
}

Published online: 11 June 2019

(c) The Author(s) 2019

\begin{abstract}
Objectives The aim was to longitudinally explore the healthcare, sickness absence (SA), and disability pension (DP) cost trajectories among newly diagnosed people with multiple sclerosis (MS), and investigate whether trajectories differ by year of MS diagnosis, sociodemographics, and multi-morbidity.

Methods People with MS in Sweden, aged 25-60 years and with a new MS diagnosis in the years 2006, 2007, 2008, or 2009 (four different cohorts) were identified in nationwide registers and followed prospectively for 5 years, determining the annual, per patient, direct (inpatient and specialised outpatient healthcare, co-payments, and dispensed drugs) and indirect (SA and DP) costs. Descriptive statistics and group-based trajectories were computed.

Results In total, 3272 people with new MS were identified. In all cohorts, direct costs increased the year after diagnosis and thereafter declined (e.g. from $€ 8261$ to $€ 9128$, and to $€ 7953,5$ years after diagnosis, for the 2006 cohort). SA costs continuously decreased over 5 years, while DP costs increased (e.g. from $€ 9795$ to $€ 2778$ vs. from $€ 7277$ to $€ 15,989$, respectively, for the 2006 cohort). When pooling all cohorts, four trajectories of direct and indirect costs were identified. A total of $32.1 \%$ of people with MS had high direct and indirect costs, which first increased and then decreased; the contrary was seen for trajectories with low direct and indirect costs.

Conclusions There is heterogeneity in the development of MS costs over time after diagnosis; decreasing cost trajectories could be associated with the use of innovative MS therapies, slowing disease progression over time.
\end{abstract}

\section{Introduction}

Through investigation of the progression of societal costs related to the healthcare resources consumed by newly diagnosed patients with chronic conditions, as well as their sickness absence (SA) or disability pension (DP), we can understand the possible progression of the burden these conditions

Electronic supplementary material The online version of this article (https://doi.org/10.1007/s41669-019-0150-3) contains supplementary material, which is available to authorized users.

Korinna Karampampa

korinna.karampampa@ki.se

1 Division of Insurance Medicine, Department of Clinical Neuroscience, Karolinska Institutet, 17177 Stockholm, Sweden

2 Institute of Health and Care Science, University of Gothenburg, SE-405 30 Gothenburg, Sweden

\section{Key Points for Decision Makers}

$32.1 \%$ of newly diagnosed people with multiple sclerosis (MS) in Sweden had high healthcare, sickness absence, and disability pension costs in the 5 years after diagnosis.

Sociodemographic factors and multi-morbidity explained part of the heterogeneity of the cost trajectories in people with MS. Disease severity and other clinical characteristics should also be explored to further understand these cost trajectories.

The year of diagnosis was significantly associated with the cost trajectories; it is, however, linked to the availability of innovative MS treatments after diagnosis, which could be associated and explain the cost progression in MS. 
impose on patients, and on countries' healthcare and social security budgets [1]. However, such information is scarce in the literature.

Multiple sclerosis (MS) is an often-disabling chronic disease and the main cause of non-traumatic neurological conditions in young adults. MS is usually diagnosed in ages 20-40 years. Sweden has the second highest prevalence of MS in Europe, estimated at 189 cases per 100,000 individuals [2].

The annual MS cost of illness from a societal perspective in Sweden was approximately 3950 million Swedish krona (SEK) (€414 million) in 2010 [3], with $75 \%$ of this cost attributed to productivity losses [3]. In addition, the cost of illness of MS has been shown to increase with disease progression [4]. While this has been recently shown by a longitudinal study in Sweden [5], most previous findings are from studies based on small, highly selected survey data of people with MS, diagnosed several years before the start of the study [6-8]. Furthermore, those studies were crosssectional; people with MS were not followed over time with regard to cost-of-illness outcomes.

The cost-of-illness trajectories over time of newly diagnosed people with MS may differ, since this group had, from the mid-1990s, access to disease-modifying therapies (DMTs; i.e. therapies after the introduction of interferonbeta), which aim to limit disease activity and presumably slow progression. In Sweden, the societal cost of illness of MS was shown to decrease a few years after diagnosis among patients with the relapsing-remitting form of MS [9]. Knowledge on whether this decrease can be expected for all newly diagnosed people with MS, whether it differs by year of MS diagnosis, and whether it is associated with sociodemographic characteristics and multi-morbidity is, however, not available. Previous studies of healthcare, SA, and DP costs for MS have shown that such outcomes are associated with age, sex, educational level, country of birth, type of living area, family situation, and morbidity $[3,10]$.

The aim of this study was to longitudinally explore the healthcare, SA, and DP cost-of-illness trajectories among newly diagnosed people with MS in Sweden, and investigate whether trajectories differed by year of MS diagnosis, sociodemographics, and multi-morbidity.

\section{Materials and Methods}

For this study, four different cohorts were established. Included were all individuals who were 25-60 years old and had their first MS diagnosis (International Classification of Diseases 10th Revision [ICD-10] code G35 [11]) according to the National Patient Register (NPR) in 2006, 2007, 2008, or 2009, and that year lived in Sweden.
The individuals in each respective cohort were followed prospectively for 5 years, including the year of inclusion. They were censored at the end of the year of emigration or death.

Microdata, from the following five nationwide registers in Sweden [12], kept by three authorities, were used in the analyses:

\section{- National Board of Health and Welfare:}

- NPR: dates and diagnoses for inpatient and specialised outpatient healthcare.

- Swedish Prescribed Drug Register (SPDR): dates, names, and costs, for all dispensed prescribed drugs.

- Cause of Death Register (CDR): year of death.

- Statistics Sweden: Longitudinal integration database for health insurance and labor market studies (LISA): sex (women/men), age, education (elementary/high school/ university or college), country of birth (Sweden/Nordic countries except Sweden/EU27 except Denmark, Finland, Iceland, and Sweden/rest of the world), type of living area (big cities/medium-sized cities/small towns or villages), family situation (married or cohabitating without children at home/married or cohabitating with children at home/single without children at home/single with children at home), indicator for receiving income from work and/or benefits during each calendar year (yes/ no).

- Swedish Social Insurance Agency: Micro data for the analysis of social insurance register (MiDAS): dates, diagnoses, and grade of SA and DP.

The registers were linked using the unique personal identity number that all residents in Sweden are assigned.

\subsection{Study Outcomes}

Healthcare resources consumed by people with MS, as well as their SA and DP, were calculated and then multiplied with unit costs (Table 1) to determine the cost of illness from a societal perspective.

Days of inpatient stays in hospitals and outpatient visits, derived from NPR, and the retrospective nationwide weights from the diagnosis-related groups (DRG) system were used to calculate the inpatient and outpatient costs. The specific DRG weight for each inpatient/outpatient use was multiplied with the cost per 1.0 DRG weight, for each year. Co-payment costs incurred by patients for the inpatient stays and outpatient visits were also estimated by summing the patient fees for inpatient and outpatient healthcare. The reimbursement period was assumed to start on 1 January, with these costs set to the value of the 
Table 1 Unit costs used in the calculation of direct and indirect costs

\begin{tabular}{|c|c|c|c|c|}
\hline & Year & $\begin{array}{l}\text { Value } \\
\text { in } 2017 \\
\mathrm{SEK}^{\mathrm{a}}\end{array}$ & $\begin{array}{l}\text { Value } \\
\text { in } 2017 \\
\text { Euros }^{\text {b }}\end{array}$ & Source \\
\hline \multirow{8}{*}{$\begin{array}{l}\text { Average inpatient and specialised outpatient cost per } \\
\text { 1.0 DRG }\end{array}$} & 2006 & 51,099 & 5303 & \multirow{8}{*}{$\begin{array}{l}\text { Swedish Association of Local Authorities and Regions, } \\
\text { cost per patient for somatic illnesses [31] }\end{array}$} \\
\hline & 2007 & 50,348 & 5226 & \\
\hline & 2008 & 51,516 & 5347 & \\
\hline & 2009 & 51,913 & 5388 & \\
\hline & 2010 & 50,582 & 5250 & \\
\hline & 2011 & 50,411 & 5232 & \\
\hline & 2012 & 49,944 & 5184 & \\
\hline & 2013 & 51,596 & 5355 & \\
\hline Co-payment for hospital stay (cost per day of stay) & 2018 & 100 & 10 & $\begin{array}{l}\text { Assume SEK100 per day, for whole Sweden, as this is } \\
\text { the case for the majority of the regions in Sweden; } \\
\text { information obtained from the Swedish Association of } \\
\text { Local Authorities and Regions. The max co-payment } \\
\text { amount for inpatient healthcare was set to SEK1500 } \\
\text { per year; assumption for whole Sweden, based on } \\
\text { information from the region Västra Götalandsre- } \\
\text { gionen [32] }\end{array}$ \\
\hline $\begin{array}{l}\text { Co-payment for specialised outpatient healthcare (cost } \\
\text { per visit) }\end{array}$ & 2018 & 273 & 28 & $\begin{array}{l}\text { The max co-payment amount for outpatient healthcare } \\
\text { was set to SEK1100 per year. Only one region in } \\
\text { Sweden has a max co-payment below SEK1100; so } \\
\text { SEK1100 was assumed for the entire country. Swed- } \\
\text { ish Association of Local Authorities and Regions [33, } \\
\text { 34] }\end{array}$ \\
\hline \multirow[t]{8}{*}{ Monthly salary including employer contributions } & 2006 & 42,061 & 4365 & \multirow{8}{*}{$\begin{array}{l}\text { The average monthly salary (2013 values) for all } \\
\text { employment types was obtained from Statistics } \\
\text { Sweden [35]. It was multiplied with the annual } \\
\text { employer contributions, available from the Swedish } \\
\text { Tax Authority [36] }\end{array}$} \\
\hline & 2007 & 41,485 & 4306 & \\
\hline & 2008 & 42,179 & 4378 & \\
\hline & 2009 & 43,000 & 4463 & \\
\hline & 2010 & 42,010 & 4360 & \\
\hline & 2011 & 41,338 & 4290 & \\
\hline & 2012 & 41,038 & 4259 & \\
\hline & 2013 & 41,284 & 4285 & \\
\hline
\end{tabular}

$D R G$ diagnosis-related groups, ECU European Currency Unit, SEK Swedish krona

${ }^{a}$ All unit costs were inflated to 2017 prices using annual Harmonised Index of Consumer Prices (HICP) for healthcare available from Eurostat [15]

${ }^{\mathrm{b}}$ The annual exchange rate for 2017 from SEK to Euros that was used was 9.6351. Source: Eurostat, Annual Exchange Rates Euro/ECU [37]

annual period co-payment ceiling in Sweden when exceeding this amount (see Table 1).

The cost of prescribed dispensed drugs was derived from SPDR, based on the pharmacy's retail price for each dispensed drug package that is reimbursed by the counties in Sweden, and the co-payment amount, paid by patients. Then the annual cost of drugs was calculated by summing all costs for dispensed drugs per individual during each year.

By adding costs of inpatient and outpatient healthcare, co-payments, and drugs, the total annual direct costs per patient were calculated.

For indirect costs, patients' productivity losses were measured based on SA and DP information, using the human capital approach [13]. All people living in Sweden with income from work or unemployment benefits are, from
16 years of age, covered by the public SA insurance system and can be granted SA benefits if their work capacity is reduced due to disease or injury. Day 1 is a waiting day, with $100 \%$ loss of income. After 7 days, a medical certificate is required. The employer usually reimburses income loss during days 2-14, after which SA benefits are administered by the Swedish Social Insurance Agency; for individuals on unemployment benefits; this happens from day 2 [14]. To prevent the introduction of bias in relation to employment status, only SA spells $>14$ days were included.

Regarding DP, all people aged 19-65 years can be granted DP if their work capacity is reduced in the long-term or permanently due to disease or injury [14].

Both SA and DP benefits can be granted for the full time $(100 \%)$ or part of the time $(75 \%, 50 \%$, or $25 \%)$ of ordinary 
work hours [14]. This means that it is possible to have both partial SA and DP concurrently, which is why we combined them in our data to calculate the net days of SA and DP. For example, two SA days at $50 \%$ were combined to one net day.

In 2012, the commonly expected age for old-age pension in Sweden was about 65 years, for both women and men.

Indirect costs were estimated based on the net days with SA and DP per year, multiplied with the age-adjusted mean salary, adding social security contributions made by employers.

All costs were inflated to 2017 prices, applying the harmonised index for consumer prices [15]. To facilitate comparability across cost-of-illness studies in Europe, costs calculated in SEK were also converted to Euros $(€)$ in 2017 values (see Table 1).

The estimated cost of illness included all costs incurred for people with MS during a calendar year, irrespective of whether MS was the main diagnosis behind the costs or not. Additionally, direct and indirect costs were calculated for when MS was the main diagnosis for the respective costs.

\subsection{Analyses}

Descriptive statistics were calculated regarding sociodemographic characteristics, multi-morbidity, and average cost of illness per patient and year for each of the four cohorts. Student's $T$ tests [16] were used to assess the significance ( $p$ value $<0.05$ ) of the cost changes over time, within each cohort, compared to baseline costs (year 1, at diagnosis), and to assess the significance of the cost differences across the four cohorts.

Then group-based cost-of-illness multi-trajectories [17] were computed summing all four study cohorts, and also stratified by study cohort. The model assumes that there are distinct groups based on the developmental trajectory of the outcomes over time, and determines the maximum likelihood of belonging to a potential trajectory group for the same individual [17].

A quadratic, zero-inflated Poisson model (please see Fig. 4 in the supplementary electronic material for more details) was used to identify trajectories with similar cost-ofillness progression, when exploring the heterogeneity in the development of cost of illness over time. To build the model utilising the Poisson distribution, direct and indirect costs were first categorised into quintiles (dataset divided in five equal parts), for each year of follow-up. The analyses were conducted using the SAS command proc traj [18].

The number of trajectories was decided based on (1) knowledge of the observed cost of illness trends using descriptive statistics; (2) size of trajectories (the size of each trajectory group) (>5\%) [17]; (3) difference in the Bayesian information criterion $(\triangle \mathrm{BIC})$-when testing models with an increasing number of trajectories, the model with the highest $2 * \Delta \mathrm{BIC}$ was selected [17]; (4) highest average posterior probabilities for belonging in the trajectory $(>0.7)$ [17]; and (5) odds of correct classification ( $>5$ ) [17].

Logistic regression [19] was used to explore sociodemographic and multi-morbidity differences between the identified cost-of-illness trajectories (covariates measured at year of diagnosis: age, sex, age at diagnosis, education, country of birth, type of living area, family situation, and multi-morbidity).

Multi-morbidity at year of diagnosis was derived utilising the Rx-Risk Comorbidity Index [20], i.e. based on the type of prescribed drugs people with MS bought, according to SPDR. Using information from the Rx-Risk Comorbidity Index, the existence of multi-morbidity (yes/no) was established, as well as whether people with MS had been diagnosed with anxiety/depression (yes/no).

The Regional Ethical Review Board of Stockholm, Sweden, approved the project.

\section{Results}

In total, in the four cohorts of people with MS first diagnosed in 2006, 2007, 2008, or 2009, respectively, 3272 individuals were included (Table 2). Except for the type of living area and family situation, there were no other statistically significant differences at year of diagnosis between the four cohorts regarding sociodemographic characteristics or multi-morbidity. Less than $1 \%$ of people with MS died within the calendar year when diagnosed; overall, $2 \%$ of all people with MS died during the follow-up.

In Table 3, the estimated cost of illness for MS is presented, over the 5-year follow-up period for all four cohorts pooled and by study cohort. The main cost driver for total costs was DP, while drug costs were the most expensive component of direct costs (see Fig. 1 in the supplementary electronic material). For all four cohorts, both direct and indirect costs increased during the year after diagnosis $(p$ value $<0.05$, compared to baseline costs, i.e. at year of diagnosis). While direct costs decreased immediately afterwards for all cohorts, indirect costs remained stable for the 2006 and 2007 cohorts for at least another year ( $p$ value $<0.05$, baseline $=$ year of diagnosis). SA costs decreased over time, while DP costs increased, indicating a shift from short-term to long-term productivity losses (see Fig. $2 \mathrm{a}$, b in the supplementary electronic material). Direct costs at years 1 and 3 and indirect costs during all years of follow-up for the 2009 cohort as well as the indirect costs at year 2 and 3 for the 2008 cohort were statistically significantly different compared to the same costs for the 2006 cohort ( $p$ value $<0.05$ ). No other statistically significant differences in the average direct and indirect cost per patient, per year were observed across the four cohorts ( $p$ value $>0.05$, baseline $=2006$ cohort ). 
Table 2 Sociodemographic and multi-morbidity characteristics at year of diagnosis, when pooling all four study cohorts and also by study cohort, which were constructed based on the year people were diagnosed with multiple sclerosis (MS)

\begin{tabular}{|c|c|c|c|c|c|c|c|}
\hline & $\begin{array}{l}\text { All cohorts } \\
N=3272\end{array}$ & $\begin{array}{l}\text { Cohort } 2006 \\
N=793\end{array}$ & $\begin{array}{l}\text { Cohort } 2007 \\
N=851\end{array}$ & $\begin{array}{l}\text { Cohort } 2008 \\
N=821\end{array}$ & $\begin{array}{l}\text { Cohort } 2009 \\
N=807\end{array}$ & $\begin{array}{l}\text { Pearson's Chi } \\
\text { square ( } p \text { value) }\end{array}$ & $\begin{array}{l}\text { Log-likelihood test } \\
\text { Chi square ( } p \text { value) }\end{array}$ \\
\hline & $N(\%)$ & $N(\%)^{\mathrm{a}}$ & $N(\%)^{\mathrm{a}}$ & $N(\%)^{\mathrm{a}}$ & $N(\%)^{\mathrm{a}}$ & & \\
\hline Sex & & & & & & $0.39(0.942)$ & $0.39(0.942)$ \\
\hline Women & $2249(68.73)$ & $552(16.87)$ & $581(17.76)$ & $563(17.21)$ & $553(16.9)$ & & \\
\hline Men & $1023(31.27)$ & $241(7.37)$ & $270(8.25)$ & $258(7.89)$ & $254(7.76)$ & & \\
\hline Age at diagnosis & & & & & & $15.48(0.079)$ & $15.57(0.076)$ \\
\hline $25-34$ & $896(27.38)$ & $216(6.6)$ & 209 (6.39) & $245(7.49)$ & $226(6.91)$ & & \\
\hline $35-44$ & $1060(32.4)$ & $247(7.55)$ & $274(8.37)$ & $257(7.85)$ & $282(8.62)$ & & \\
\hline $45-54$ & $850(25.98)$ & $203(6.2)$ & $231(7.06)$ & $212(6.48)$ & $204(6.23)$ & & \\
\hline $55-60$ & $466(14.24)$ & $127(3.88)$ & $137(4.19)$ & $107(3.27)$ & $95(2.9)$ & & \\
\hline Educational level $^{\mathrm{b}}$ & & & & & & $7.74(0.56)$ & $7.94(0.54)$ \\
\hline Elementary (0-9 years) & $433(13.60)$ & $95(2.98)$ & $110(3.45)$ & $112(3.52)$ & $116(3.64)$ & & \\
\hline High school (10-12 years) & $1561(49.03)$ & $400(12.56)$ & $419(13.16)$ & $380(11.93)$ & $362(11.37)$ & & \\
\hline $\begin{array}{l}\text { University/college } \\
\text { (>12 years })\end{array}$ & $1190(37.37)$ & $291(9.14)$ & $310(9.74)$ & $299(9.39)$ & $290(9.11)$ & & \\
\hline Country of birth ${ }^{\mathrm{b}}$ & & & & & & $12.73(0.389)$ & $13.16(0.358)$ \\
\hline Sweden & $2826(88.76)$ & $708(22.24)$ & $733(23.02)$ & $700(21.98)$ & $685(21.51)$ & & \\
\hline $\begin{array}{l}\text { Nordic countries (except } \\
\text { Sweden) }\end{array}$ & $85(2.67)$ & $13(0.41)$ & $34(1.07)$ & $19(0.6)$ & $19(0.6)$ & & \\
\hline $\begin{array}{l}\text { EU27 (except Denmark, Fin- } \\
\text { land, Iceland, and Sweden) }\end{array}$ & $71(2.23)$ & $19(0.6)$ & $18(0.57)$ & $17(0.53)$ & $17(0.53)$ & & \\
\hline Rest of the world & $202(6.34)$ & $46(1.44)$ & $54(1.7)$ & $55(1.73)$ & $47(1.48)$ & & \\
\hline Type of living area ${ }^{\mathrm{b}}$ & & & & & & $13.73(0.033)$ & $13.94(0.03)$ \\
\hline Big cities & $1200(37.69)$ & $311(9.77)$ & $277(8.7)$ & $310(9.74)$ & $302(9.48)$ & & \\
\hline Medium-sized cities & $1087(34.14)$ & $268(8.42)$ & $307(9.64)$ & $248(7.79)$ & $264(8.29)$ & & \\
\hline Small towns/villages & $897(28.17)$ & $207(6.5)$ & $255(8.01)$ & $233(7.32)$ & $202(6.34)$ & & \\
\hline Family situation $^{\mathrm{b}}$ & & & & & & $34.24(<0.0001)$ & $34.68(<0.0001)$ \\
\hline $\begin{array}{l}\text { Married/cohabitating with- } \\
\text { out children at home }\end{array}$ & $395(12.41)$ & $129(4.05)$ & $117(3.67)$ & $85(2.67)$ & $64(2.01)$ & & \\
\hline $\begin{array}{l}\text { Married/cohabitating with } \\
\text { children at home }\end{array}$ & $1320(41.46)$ & $308(9.67)$ & $361(11.34)$ & $333(10.46)$ & $318(9.99)$ & & \\
\hline $\begin{array}{l}\text { Single without children at } \\
\text { home }\end{array}$ & $1166(36.62)$ & $270(8.48)$ & $279(8.76)$ & $305(9.58)$ & $312(9.8)$ & & \\
\hline Single with children at home & $303(9.52)$ & $79(2.48)$ & $82(2.58)$ & $68(2.14)$ & $74(2.32)$ & & \\
\hline Multi-morbidity & & & & & & $4.77(0.19)$ & $4.96(0.175)$ \\
\hline Yes & $3027(92.51)$ & $730(22.31)$ & $786(24.02)$ & $751(22.95)$ & $760(23.23)$ & & \\
\hline No & 245 (7.49) & $63(1.93)$ & $65(1.99)$ & $70(2.14)$ & $47(1.44)$ & & \\
\hline Anxiety/depression & & & & & & $5.64(0.131)$ & $5.57(0.135)$ \\
\hline Yes & $181(5.53)$ & $55(1.68)$ & $50(1.53)$ & $40(1.22)$ & $36(1.1)$ & & \\
\hline No & $3091(94.47)$ & $738(22.56)$ & $801(24.48)$ & $781(23.87)$ & $771(23.56)$ & & \\
\hline
\end{tabular}

${ }^{\mathrm{a}}$ The percentages are calculated as $n$ divided with the total number of individuals in the study population (3272 if not otherwise indicated; see ${ }^{\mathrm{b}}$ ) ${ }^{\mathrm{b}}$ The total number of individuals with this type of information in the study population (i.e. all individuals excluding those with missing information in this category) was 3184

When pooling all cohorts, four trajectories of costs were identified. The largest trajectory included $32.1 \%$ of the people with MS and was characterised with "high direct and indirect costs" (the term "high" is chosen to give an indication of how costs are scaled in comparison with the other trajectories where direct and/or indirect costs were lower), while $19.9 \%$ of people with MS belonged to the trajectory of "low direct and indirect costs" (similarly, "low" denotes the comparison between the observed trajectories). The 
Table 3 Mean costs per patient per year [ $95 \%$ confidence intervals] in Euros 2017, by all costs and by costs generated with multiple sclerosis (MS) as the main diagnosis, when pooling all four study cohorts and also by study cohort, and by type of cost [(a) Direct costs, $(b)$ Indirect costs]

\begin{tabular}{|c|c|c|c|c|c|}
\hline & $\begin{array}{l}\text { All cohorts } \\
N=3272\end{array}$ & $\begin{array}{l}\text { Cohort } 2006 \\
N=793\end{array}$ & $\begin{array}{l}\text { Cohort } 2007 \\
N=851\end{array}$ & $\begin{array}{l}\text { Cohort } 2008 \\
N=821\end{array}$ & $\begin{array}{l}\text { Cohort } 2009 \\
N=807\end{array}$ \\
\hline \multicolumn{6}{|c|}{ (a) Direct costs } \\
\hline \multicolumn{6}{|c|}{ Inpatient costs } \\
\hline Year 1 & $2642[2121-3163]$ & 2748 [2218-3277] & $2439[2038-2841]$ & $3210[2531-3890]$ & 2172 [1698-2645] \\
\hline Year 2 & 1574 [1122-2026] & $1693[1155-2230]$ & 1617 [1187-2047] & $1463[1145-1780]^{*}$ & $1524[1000-2048]$ \\
\hline Year 3 & $1606[1250-1963]$ & $1710[1285-2134]$ & $1672[1331-2013]$ & $1721[1330-2113]$ & $1322[1052-1592]$ \\
\hline Year 4 & $1488[1076-1900]$ & 1305 [998-1611] & 1745 [1090-2399] & $1531[1205-1856]$ & $1372[1012-1732]$ \\
\hline Year 5 & 1515 [1159-1872] & $1672[1312-2032]$ & 1345 [1064-1626] & $1544[1127-1960]$ & 1500 [1132-1869] \\
\hline \multicolumn{6}{|c|}{ Inpatient costs with MS as main diagnosis } \\
\hline Year 1 & $664[510-819]$ & $823[658-988]$ & 600 [464-737] & $803[574-1032]$ & $431[344-519]$ \\
\hline Year 2 & $492[302-683]$ & $366[224-508]$ & $567[345-789]$ & $571[352-790]$ & $465[288-643]$ \\
\hline Year 3 & $585[363-807]$ & $525[300-749]$ & 699 [442-957] & 709 [462-956] & $406[246-567]$ \\
\hline Year 4 & 494 [291-696] & 389 [203-575] & $699[447-951]$ & $553[343-764]$ & $333[171-495]$ \\
\hline Year 5 & $458[263-654]$ & $522[292-751]$ & $519[315-724]$ & 468 [259-677] & $323[184-463]$ \\
\hline \multicolumn{6}{|c|}{ Outpatient costs } \\
\hline Year 1 & $1768[1655-1880]$ & $1746[1605-1887]$ & $1481[1383-1579]$ & $1720[1625-1814]$ & 2124 [2007-2241] \\
\hline Year 2 & $1235[1128-1341]$ & $1004[886-1121]$ & $1130[1049-1210]$ & $1461[1341-1582]$ & $1343[1235-1451]$ \\
\hline Year 3 & 1234 [1124-1344] & 1007 [912-1103] & $1223[1116-1331]$ & $1216[1107-1325]$ & 1488 [1361-1616] \\
\hline Year 4 & $1266[1157-1375]$ & 1128 [1016-1240] & 1017 [920-1115] & $1426[1314-1538]$ & 1492 [1376-1607] \\
\hline Year 5 & 1318 [1200-1436] & 1059 [941-1177] & $1222[1120-1324]$ & 1575 [1451-1699] & 1415 [1286-1544] \\
\hline \multicolumn{6}{|c|}{ Outpatient costs with MS as main diagnosis } \\
\hline Year 1 & 764 [714-813] & 768 [707-829] & 627 [591-664] & 752 [706-798] & 907 [852-962] \\
\hline Year 2 & $626[572-680]$ & 480 [439-522] & 618 [570-666] & 699 [637-760] & 706 [642-770] \\
\hline Year 3 & 599 [538-660] & 467 [420-514] & 587 [530-644] & 577 [515-639] & 764 [686-843] \\
\hline Year 4 & 616 [553-679] & 474 [428-519] & 516 [456-576] & 691 [624-757] & 784 [705-864] \\
\hline Year 5 & $661[585-736]$ & $472[400-544]$ & 628 [559-697] & 824 [739-909] & 718 [642-793] \\
\hline \multicolumn{6}{|c|}{ Co-payments } \\
\hline Year 1 & 113 [109-117] & 115 [111-119] & 110 [106-114] & 116 [112-120] & 112 [108-115] \\
\hline Year 2 & 77 [73-81] & 78 [74-82] & $76[72-80]$ & 78 [74-82] & 77 [73-81] \\
\hline Year 3 & $70[66-74]$ & 69 [65-73] & 65 [62-69] & 70 [66-74] & 75 [72-79] \\
\hline Year 4 & $68[64-71]$ & 63 [59-66] & $62[58-65]$ & $72[68-76]$ & $73[69-76]$ \\
\hline Year 5 & 69 [65-73] & 63 [59-68] & $68[64-72]$ & 73 [69-77] & $70[66-74]$ \\
\hline \multicolumn{6}{|c|}{ All drug costs } \\
\hline Year 1 & 4058 [3707-4409] & 3652 [3302-4003] & 3637 [3304-3971] & 4221 [3865-4577] & $4721[4356-5086]$ \\
\hline Year 2 & 6770 [6291-7250] & 6353 [5872-6835] & 6613 [6139-7088] & $6863[6365-7362]$ & 7250 [6787-7713] \\
\hline Year 3 & 6117 [5646-6589] & 6066 [5543-6588] & $6263[5800-6726]$ & 5758 [5313-6203] & 6382 [5926-6838] \\
\hline Year 4 & 5655 [5193-6118] & 5485 [4989-5981] & 5933 [5483-6384] & 5272 [4834-5710] & 5929 [5464-6395] \\
\hline Year 5 & $5298[4836-5761]$ & 5158 [4668-5649] & 5292 [4863-5722] & 5181 [4718-5645] & 5561 [5094-6028] \\
\hline \multicolumn{6}{|c|}{ MS drug costs } \\
\hline Year 1 & 3614 [3270-3957] & 3198 [2854-3541] & 3203 [2876-3530] & 3729 [3386-4072] & 4325 [3964-4686] \\
\hline Year 2 & 6220 [5753-6687] & 5812 [5337-6286] & 6063 [5591-6535] & 6266 [5801-6731] & 6739 [6281-7196] \\
\hline Year 3 & $5528[5071-5984]$ & 5378 [4901-5855] & 5669 [5211-6126] & $5230[4785-5674]$ & $5833[5385-6281]$ \\
\hline Year 4 & 5042 [4596-5487] & 4757 [4307-5207] & 5307 [4866-5749] & $4673[4244-5101]$ & 5429 [4968-5891] \\
\hline Year 5 & 4647 [4207-5087] & 4381 [3949-4812] & $4648[4225-5072]$ & 4492 [4047-4938] & 5066 [4606-5527] \\
\hline \multicolumn{6}{|c|}{ Total direct costs } \\
\hline Year 1 & 8581 [7926-9237] & 8261 [7565-8956] & 7668 [7141-8195] & $9267[8475-10,060]$ & 9128 [8521-9736] \\
\hline Year 2 & 9655 [8996-10,316] & 9128 [8396-9860] & $9435[8826-10,045]$ & $9864[9289-10,440]$ & $10,194[9472-10,917]$ \\
\hline Year 3 & 9027 [8434-9621] & 8852 [8172-9532] & 9224 [8656-9792] & 8765 [8179-9351] & 9268 [8728-9808] \\
\hline
\end{tabular}


Table 3 (continued)

\begin{tabular}{|c|c|c|c|c|c|}
\hline & $\begin{array}{l}\text { All cohorts } \\
N=3272\end{array}$ & $\begin{array}{l}\text { Cohort } 2006 \\
N=793\end{array}$ & $\begin{array}{l}\text { Cohort } 2007 \\
N=851\end{array}$ & $\begin{array}{l}\text { Cohort } 2008 \\
N=821\end{array}$ & $\begin{array}{l}\text { Cohort } 2009 \\
N=807\end{array}$ \\
\hline Year 4 & 8476 [7843-9109] & $7980[7382-8578]$ & 8757 [7972-9543] & $8301[7736-8865]$ & 8865 [8280-9451] \\
\hline Year 5 & 8200 [7599-8801] & $7953[7341-8565]$ & 7927 [7405-8449] & $8373[7728-9018]$ & 8546 [7920-9171] \\
\hline \multicolumn{6}{|c|}{ Total direct costs with MS as main diagnosis } \\
\hline Year 1 & $5155[4760-5550]$ & $4903[4492-5314]$ & $4541[4179-4903]$ & $5401[4980-5822]$ & $5775[5389-6161]$ \\
\hline Year 2 & 7415 [6914-7917] & $6736[6233-7239]$ & 7323 [6807-7839] & 7614 [7111-8118] & 7987 [7503-8471] \\
\hline Year 3 & $6781[6282-7281]$ & $6439[5915-6962]$ & $7021[6511-7531]$ & $6586[6100-7072]$ & 7079 [6600-7557] \\
\hline Year 4 & 6219 [5733-6704] & $5682[5191-6173]$ & $6584[6097-7071]$ & 5989 [5515-6463] & 6619 [6130-7109] \\
\hline Year 5 & $5834[5348-6320]$ & 5438 [4957-5919] & $5864[5396-6331]$ & $5857[5350-6365]$ & $6177[5689-6665]$ \\
\hline \multicolumn{6}{|c|}{ (b) Indirect costs } \\
\hline \multicolumn{6}{|l|}{ SA costs ${ }^{\mathrm{a}}$} \\
\hline Year 1 & 8458 [7407-9510] & $9795[8743-10,847]$ & 8684 [7741-9627] & 8532 [7541-9523] & $7485[6562-8408]$ \\
\hline Year 2 & $6065[5179-6950]$ & $9610[8483-10,736]$ & $8588[7566-9611]$ & 8175 [7118-9232] & $7460[6459-8462]$ \\
\hline Year 3 & $4546[3789-5303]$ & $7811[6758-8864]$ & $5591[4782-6400]$ & $5010[4216-5804]$ & $5846[4960-6731]$ \\
\hline Year 4 & $3881[3181-4580]$ & $5604[4704-6505]$ & $3201[2612-3790]$ & $4531[3770-5292]$ & $4847[4071-5623]$ \\
\hline Year 5 & & 2778 [2206-3349] & $3561[2880-4241]$ & 4240 [3497-4984] & $4944[4142-5746]$ \\
\hline \multicolumn{6}{|c|}{ SA costs with MS as main diagnosis ${ }^{a}$} \\
\hline Year 1 & 2508 [1980-3037] & $2591[2034-3148]$ & $2638[2125-3152]$ & 2458 [1918-2997] & $2345[1841-2850]$ \\
\hline Year 2 & 3689 [2964-4415] & 3957 [3190-4724] & 3757 [3037-4477] & 3301 [2617-3986] & $3742[3011-4474]$ \\
\hline Year 3 & 3227 [2560-3893] & 3912 [3127-4697] & 2912 [2326-3498] & 2755 [2153-3358] & $3327[2635-4020]$ \\
\hline Year 4 & 2830 [2216-3443] & 3071 [2377-3765] & $2327[1813-2841]$ & $2746[2141-3351]$ & 3174 [2533-3815] \\
\hline Year 5 & 2606 [2013-3199] & 1817 [1327-2306] & 2538 [1946-3131] & 2755 [2154-3356] & $3312[2623-4001]$ \\
\hline \multicolumn{6}{|l|}{$\mathrm{DP}$ costs } \\
\hline Year 1 & $6972[5836-8108]$ & 7277 [6107-8447] & 8055 [6888-9222] & $6583[5466-7699]$ & $5974[4884-7065]$ \\
\hline Year 2 & 8691 [7465-9917] & $9375[8093-10,658]$ & $10,232[8965-11,500]$ & 8259 [7038-9479] & $6897[5762-8032]$ \\
\hline Year 3 & $10,941[9609-12,274]$ & $12,028[10,618-13,437]$ & $13,297[11,876-14,718]$ & $10,548[9235-11,862]$ & $7891[6705-9077]$ \\
\hline Year 4 & $12,534[11,150-13,918]$ & $14,412[12,919-15,904]$ & $14,923[13,484-16,362]$ & $11,519[10,175-12,863]$ & $9281[8020-10,541]$ \\
\hline Year 5 & $13,462[12,054-14,870]$ & $15,989[14,473-17,506]$ & $14,987[13,563-16,412]$ & $12,460[11,075-13,845]$ & $10,410[9106-11,715]$ \\
\hline \multicolumn{6}{|c|}{ DP costs with MS as main diagnosis } \\
\hline Year 1 & $1731[1175-2287]$ & $1738[1152-2325]$ & $2569[1877-3262]$ & $1936[1339-2534]$ & $680[331-1028]$ \\
\hline Year 2 & 3321 [2559-4082] & 3443 [2646-4239] & $4454[3585-5324]$ & 3490 [2697-4283] & 1895 [1309-2481] \\
\hline Year 3 & $5355[4387-6323]$ & $5545[4536-6553]$ & $6866[5786-7946]$ & 5737 [4737-6737] & $3272[2489-4056]$ \\
\hline Year 4 & $6947[5866-8028]$ & $7458[6319-8598]$ & 8654 [7478-9829] & $6872[5799-7945]$ & $4802[3866-5739]$ \\
\hline Year 5 & 7951 [6806-9097] & $8954[7723-10,186]$ & $9179[7987-10,371]$ & $7841[6691-8992]$ & $5831[4824-6838]$ \\
\hline \multicolumn{6}{|c|}{ Total indirect costs } \\
\hline Year 1 & $15,596[14,248-16,945]$ & $17,072[15,667-18,477]$ & $16,739[15,399-18,080]$ & $15,114[13,773-16,456]$ & $13,459[12,154-14,765]$ \\
\hline Year 2 & $17,149[15,676-18,622]$ & $18,985[17,462-20,508]$ & $18,820[17,330-20,310]$ & $16,434[14,962-17,905]$ & $14,358[12,950-15,765]$ \\
\hline Year 3 & $17,006[15,536-18,475]$ & $19,838[18,276-21,401]$ & $18,888[17,378-20,397]$ & $15,559[14,127-16,990]$ & $13,737[12,361-15,113]$ \\
\hline Year 4 & $17,080[15,611-18,548]$ & $20,016[18,419-21,614]$ & $18,124[16,660-19,587]$ & $16,050[14,609-17,491]$ & $14,128[12,755-15,501]$ \\
\hline Year 5 & $17,343[15,875-18,810]$ & $18,767[17,243-20,290]$ & $18,548[17,079-20,017]$ & $16,700[15,251-18,150]$ & $15,355[13,927-16,783]$ \\
\hline \multicolumn{6}{|c|}{ Total indirect costs with MS as main diagnosis } \\
\hline Year 1 & 4239 [3480-4998] & 4329 [3536-5123] & 5208 [4369-6047] & 4394 [3601-5186] & 3025 [2415-3635] \\
\hline Year 2 & 7010 [5985-8035] & 7400 [6323-8477] & 8211 [7128-9294] & 6791 [5776-7806] & 5637 [4712-6562] \\
\hline Year 3 & 8582 [7437-9727] & 9457 [8220-10,693] & $9778[8590-10,966]$ & 8493 [7352-9634] & $6600[5586-7613]$ \\
\hline Year 4 & 9777 [8566-10,987] & $10,530[9230-11,829]$ & $10,981[9736-12,226]$ & $9619[8419-10,818]$ & 7976 [6877-9076] \\
\hline Year 5 & $10,557[9303-11,811]$ & $10,771[9486-12,056]$ & $11,717[10,427-13,008]$ & $10,596[9347-11,846]$ & 9142 [7953-10,332] \\
\hline
\end{tabular}

$D P$ disability pension, $S A$ sickness absence

${ }^{\mathrm{a}}$ In order not to introduce bias in relation to employment status, only SA spells > 14 days were included in the SA costs calculation 


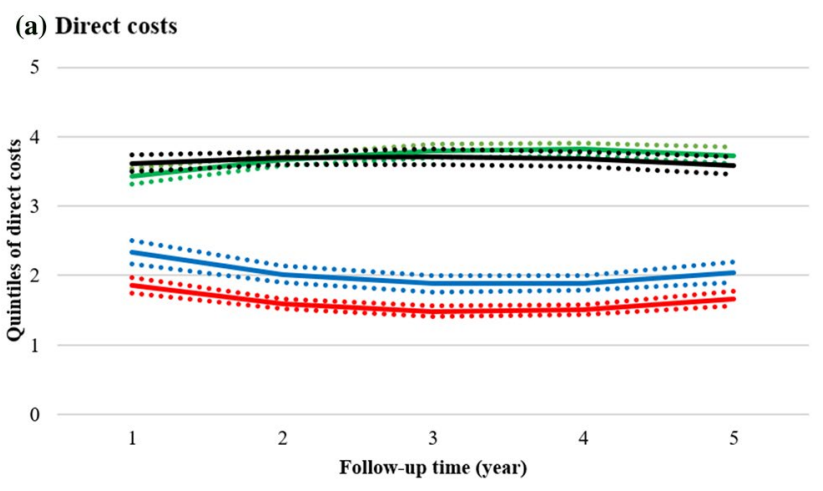

(b) Indirect costs

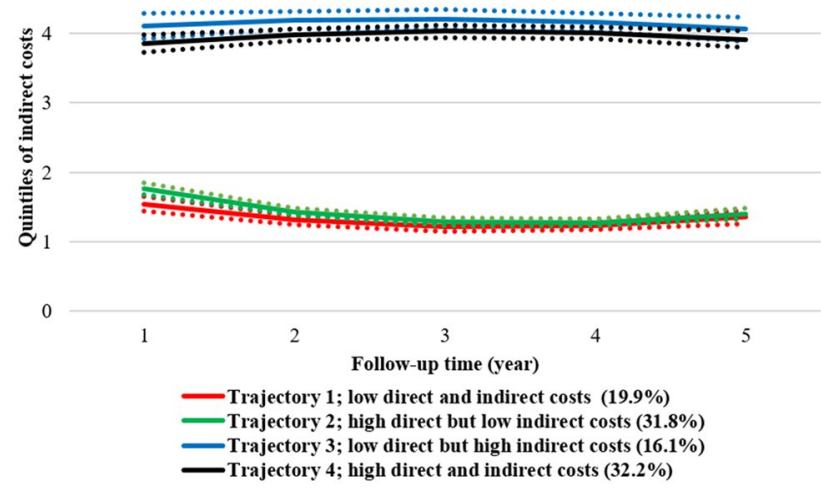

Fig. 1 Estimated direct and indirect cost trajectories, overall for all cohorts (pooled data from all four cohorts; a direct costs, $\mathbf{b}$ indirect costs) during the 5-year follow-up. Dotted lines present the $95 \%$ confidence intervals. Note 1: The values $0-1$ on the $y$-axis denote the 1 st quintile ( $0-20 \%$ of observations), $1-2$ the 2 nd (20-40\% of observations), $2-3$ the 3 rd (40-60\% of observations), $3-4$ the 4 th $(60-80 \%$ of observations), and $4-5$ the 5 th (80-100\% of observations). Each included individual with MS is classified for each year of follow-up into the respective quintile for direct and indirect costs, i.e. individuals can be classified in different quintiles for each year of follow-up. For example, an individual diagnosed with MS in 2006 may be in the 2nd quintile (between $20 \%$ and $40 \%$ of observations) in 2006 when it comes to direct costs, but in the 3rd quintile regarding indirect costs. Note 2: Trajectories represent clusters (sub-groups) of people with MS that have a similar likelihood for belonging to the identified quintiles of costs, over time (up to 5 years after diagnosis). $M S$ multiple sclerosis

other two trajectories were "high direct, but low indirect costs", to which $31.8 \%$ of people with MS belonged, and "low direct, but high indirect costs", with $16.1 \%$ of people with MS (Fig. 1). Costs in trajectories with high direct costs first increased and then decreased over time; the contrary was seen for trajectories with low direct and indirect costs.

From the trajectory analysis, no significant cohort effects were observed since similar cost trajectories were found for the four cohorts separately, except that the majority of people with MS in the 2009 cohort had high direct but low indirect costs, while only the next largest trajectory had both high direct and indirect costs compared to the trajectories observed for the three earlier cohorts (2006, 2007, and 2008). As an example of our findings, direct and indirect cost trajectories for the 2006 and 2009 cohorts are shown in Fig. 2; results for the remaining cohorts (2007 and 2008) are presented in the supplementary electronic material, Fig. 3.

Between the trajectories, several differences in sociodemographic and multi-morbidity characteristics at year of diagnosis were identified (Table 4). There were more women in the trajectories with high direct costs; these trajectories also had most people with MS diagnosed at a young age (less than 45 years of age), and people with MS with at least high school education; the most prominent country of birth was Sweden, and most individuals lived in big cities at the time of diagnosis. The trajectory "high direct and indirect costs" had the largest proportion of people with MS with multi-morbidity at time of diagnosis.

\section{Discussion}

In this register-based prospective cohort study, we explored, through group-based multi-trajectory modelling, the heterogeneity in the development of the cost of illness over time among newly diagnosed MS patients, and investigated whether cost-of-illness trajectories differ by year of MS diagnosis, sociodemographics, and multi-morbidity. Our findings suggest that there were sociodemographic and multi-morbidity differences between trajectories. There were no significant differences in the trajectories between cohorts, confirming our findings in each of the four cohorts and indicating that the cost of illness may be predominantly connected with disease activity and treatment with DMTs.

For $63.9 \%$ of people with MS, direct costs increased 1 year after diagnosis and thereafter decreased. A similar result has been reported in a previous study in Sweden investigating cost-of-illness trajectories of newly diagnosed patients with the relapsing-remitting form of the disease [9]. The presence of high disease activity at the time of MS diagnosis, possibly leading to other somatic or mental problems, such as anxiety/depression, etc., could explain the high MS cost of illness during the year the MS diagnosis was received, since MS costs are higher with higher MS severity and for MS relapses [4, 7, 21, 22]. This initial increase in direct costs could also be due to diagnostic procedures and the need for more healthcare visits during the first year of diagnosis. In 2005, a change in the diagnostic procedures for MS was initiated-guidelines were revised in order to enable diagnosis of people with MS at their first clinical episode and using diagnostic laboratory findings [23]. Therefore, an increasing number of comprehensive diagnostic procedures may have been performed during the first year when diagnosed with MS, leading to high cost of MS during that year. 
2a-b: cohort 2006

(a) Direct costs

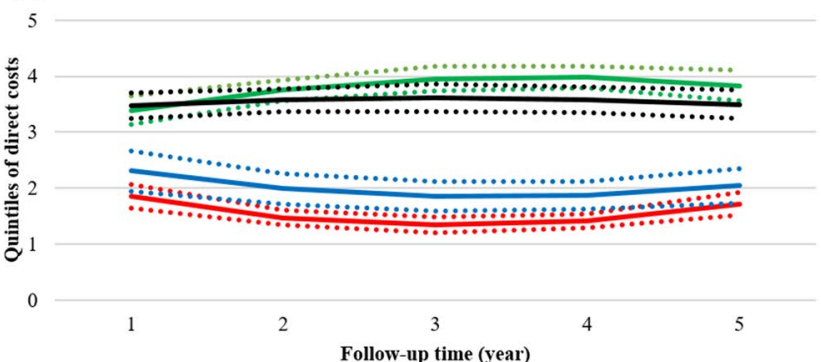

(b) Indirect costs

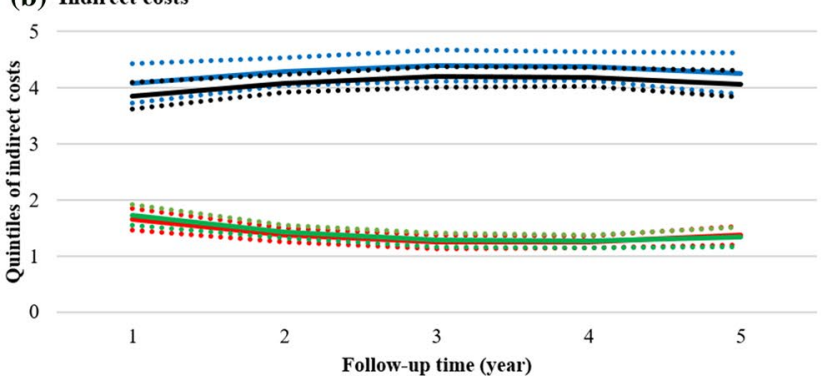

-T rajectory $1 ;$ low direct and ind irect costs $(22.1 \%$

-T rajectory 2; high direct but low ind irect costs $(25.1 \%)$

- Trajectory 3; low direct but high ind irect costs $(17.2 \%$ )

- Trajectory 4; high direct and ind irect costs (35.5\%)

Fig. 2 Estimated direct and indirect cost trajectories for the 2006 (a direct costs, b indirect costs) and 2009 (c direct costs, d indirect costs) study cohorts, separately, during the 5-year follow-up. Dotted lines present the $95 \%$ confidence intervals. Note 1 : The values $0-1$ on the $y$-axis denote the 1st quintile (0-20\% of observations), $1-2$ the 2nd (20-40\% of observations), $2-3$ the 3 rd (40-60\% of observations), $3-4$ the 4 th (60-80\% of observations), and $4-5$ the 5 th $(80-100 \%$ of observations). Each included individual with MS is classified for each year of follow-up into the respective quintile for direct and indirect

We also found that the main cost driver for direct costs was dispensed prescription drugs, in particular, MS DMTs. As three previous studies have shown [4, 24, 25], in the last 2 decades, drugs have become one of the main cost drivers in MS; new DMTs have changed the treatment landscape of MS, decreasing the need for expensive inpatient healthcare [22]. This change could also be linked to the fact that from the 2007 cohort, direct costs were higher for each cohortan increase in the proportion of people with MS receiving MS treatment with DMTs directly after MS diagnosis could lead to the observed result. It is plausible that the need for healthcare visits among people with MS decreases after MS diagnosis and when DMTs start inhibiting relapses, resulting in lower direct costs.

Combining the other two smaller trajectories (with fewer people with MS), $36.1 \%$ had direct costs that decreased in the year after diagnosis, and later increased. The people with MS in these two trajectories were slightly older compared to those in the other two larger trajectories. More research about the mechanisms behind this is warranted.

\section{2c-d: cohort 2009}

(c) Direct costs

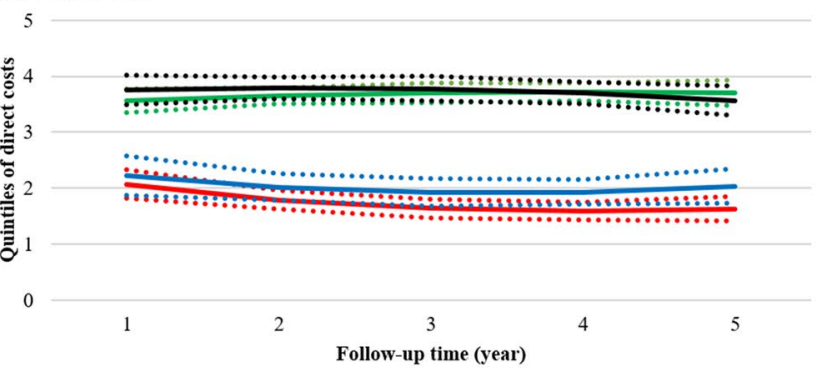

(d) Indirect costs

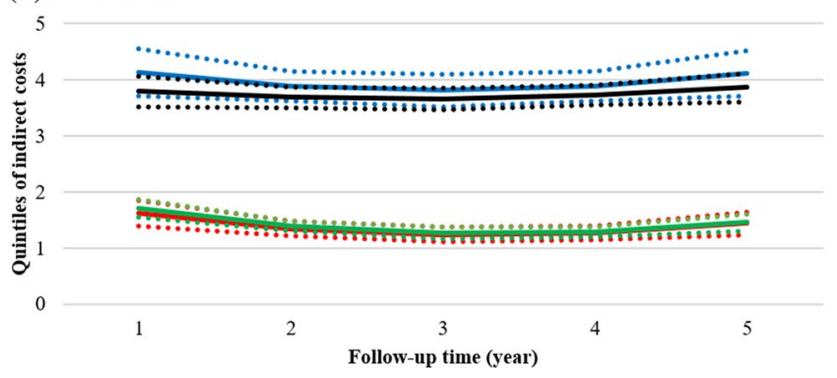

-T rajectory 1 ; low direct and ind irect costs $(18.7 \%)$

- Trajectory 2; high direct but low ind irect costs $(39.1 \%$

- Trajectory 3; low direct but high ind irect cost (13.2\%)

Trajectory 4; high direct and ind irect costs (29\%)

costs, i.e. individuals can be classified in different quintiles for each year of follow-up. For example, an individual diagnosed with MS in 2006 may be in the 2nd quintile (between $20 \%$ and $40 \%$ of observations) in 2006 when it comes to direct costs, but in the 3rd quintile regarding indirect costs. Note 2: Trajectories represent clusters (subgroups) of people with MS that have a similar likelihood for belonging to the identified quintiles of costs, over time (up to 5 years after diagnosis). $M S$ multiple sclerosis

One explanation could, for example, be that they had their diagnosis very late after MS onset and symptoms, so they had more advanced MS at diagnosis and, consequently, did not require so many diagnostic procedures to establish the diagnosis, hence, not leading to an initial increase of their direct costs as compared to the other two trajectories.

For $48.2 \%$ of people with MS, indirect costs first increased then declined afterwards. As with the interpretation of the changes in direct costs over time, the use of MS DMTs could have played a significant role in the decline in indirect costs as well, with earlier use of DMTs slowing the accumulation of MS disability [26], hence, delaying/reducing the need for SA and DP.

Another possible explanation that needs to be considered is the general decline in the number of SA and DP days in the population in Sweden in the studied years, until 2010, and a slight increase thereafter [14]. Those changes, at the population level, are related to changes in the SA rules, introducing stricter criteria for SA and DP benefits. 
Table 4 Sociodemographic and multi-morbidity differences across the estimated four trajectories of newly diagnosed individuals with multiple sclerosis (MS)

\begin{tabular}{|c|c|c|c|c|c|c|c|}
\hline & $\begin{array}{l}\text { Trajectory } 1: \\
\text { low direct and } \\
\text { indirect costs }\end{array}$ & $\begin{array}{l}\text { Trajectory 2: } \\
\text { high direct but } \\
\text { low indirect } \\
\text { costs }\end{array}$ & $\begin{array}{l}\text { Trajectory } 3: \text { low } \\
\text { direct but high } \\
\text { indirect costs }\end{array}$ & $\begin{array}{l}\text { Trajectory 4: } \\
\text { high direct and } \\
\text { indirect costs }\end{array}$ & $\begin{array}{l}\text { Pearson's Chi } \\
\text { square ( } p \text { value) }\end{array}$ & $\begin{array}{l}\text { Log-likelihood } \\
\text { test Chi square } \\
(p \text { value })\end{array}$ & Diff. in $\mathrm{R} 2^{\mathrm{C}}$ \\
\hline & $19.9 \%$ & $31.8 \%$ & $16.1 \%$ & $32.1 \%$ & & & \\
\hline & $N(\%)^{\mathrm{a}}$ & $N(\%)^{\mathrm{a}}$ & $N(\%)^{\mathrm{a}}$ & $N(\%)^{\mathrm{a}}$ & & & \\
\hline Sex & & & & & $37.81(<0.0001)$ & $37.98(<0.0001)$ & 0.024 \\
\hline Women & $410(12.55)$ & $678(20.76)$ & $362(11.08)$ & $797(24.4)$ & & & \\
\hline Men & $252(7.72)$ & $351(10.75)$ & $148(4.53)$ & $268(8.21)$ & & & \\
\hline Age at diagnosis & & & & & $\begin{array}{l}368.49 \\
\quad(<0.0001)\end{array}$ & $\begin{array}{l}373.23 \\
\quad(<0.0001)\end{array}$ & 0.050 \\
\hline $25-34$ & $179(5.47)$ & $424(12.96)$ & $56(1.71)$ & $237(7.24)$ & & & \\
\hline $35-44$ & $202(6.17)$ & $375(11.46)$ & $108(3.3)$ & $375(11.46)$ & & & \\
\hline $45-54$ & $181(5.53)$ & $173(5.29)$ & $193(5.9)$ & $303(9.26)$ & & & \\
\hline $55-60$ & $101(3.09)$ & $57(1.74)$ & $153(4.68)$ & $155(4.74)$ & & & \\
\hline $\begin{array}{l}\text { Educational } \\
\text { level }^{\mathrm{b}}\end{array}$ & & & & & $\begin{array}{l}157.85 \\
\quad(<0.0001)\end{array}$ & $\begin{array}{l}159.62 \\
\quad(<0.0001)\end{array}$ & 0.018 \\
\hline $\begin{array}{l}\text { Elementary } \\
\text { (0-9 years) }\end{array}$ & $78(2.45)$ & $86(2.7)$ & $113(3.55)$ & $156(4.9)$ & & & \\
\hline $\begin{array}{l}\text { High school } \\
\text { (10-12 years) }\end{array}$ & $267(8.39)$ & $440(13.82)$ & $272(8.54)$ & $582(18.28)$ & & & \\
\hline $\begin{array}{l}\text { University/ } \\
\text { college } \\
\text { (>12 years) }\end{array}$ & $295(9.27)$ & $454(14.26)$ & $119(3.74)$ & $322(10.11)$ & & & \\
\hline Country of birth ${ }^{b}$ & & & & & $11.07(0.5229)$ & $11.47(0.4888)$ & -0.001 \\
\hline Sweden & $559(17.56)$ & $868(27.26)$ & $459(14.42)$ & $940(29.52)$ & & & \\
\hline $\begin{array}{l}\text { Nordic coun- } \\
\text { tries (except } \\
\text { Sweden) }\end{array}$ & $17(0.53)$ & $26(0.82)$ & $14(0.44)$ & $28(0.88)$ & & & \\
\hline $\begin{array}{l}\text { EU27 (except } \\
\text { Denmark, } \\
\text { Finland, } \\
\text { Iceland, and } \\
\text { Sweden) }\end{array}$ & $19(0.6)$ & $23(0.72)$ & $<10(0.25)$ & $21(0.66)$ & & & \\
\hline $\begin{array}{l}\text { Rest of the } \\
\text { world }\end{array}$ & $45(1.41)$ & $63(1.98)$ & $23(0.72)$ & $71(2.23)$ & & & \\
\hline $\begin{array}{l}\text { Type of living } \\
\text { area }^{\text {b }}\end{array}$ & & & & & $39.48(<0.0001)$ & $40.05(<0.0001)$ & 0.007 \\
\hline Big cities & $266(8.35)$ & $419(13.16)$ & $144(4.52)$ & $371(11.65)$ & & & \\
\hline $\begin{array}{l}\text { Medium-sized } \\
\text { cities }\end{array}$ & $211(6.63)$ & $322(10.11)$ & $191(6)$ & $363(11.4)$ & & & \\
\hline $\begin{array}{l}\text { Small towns/ } \\
\text { villages }\end{array}$ & $163(5.12)$ & $239(7.51)$ & $169(5.31)$ & $326(10.24)$ & & & \\
\hline Family situation $^{\mathrm{b}}$ & & & & & $\begin{array}{l}109.10 \\
\quad(<0.0001)\end{array}$ & $\begin{array}{l}112.09 \\
\quad(<0.0001)\end{array}$ & 0.005 \\
\hline $\begin{array}{l}\text { Married/ } \\
\text { cohabitat- } \\
\text { ing without } \\
\text { children at } \\
\text { home }\end{array}$ & $83(2.61)$ & $69(2.17)$ & $104(3.27)$ & $139(4.37)$ & & & \\
\hline $\begin{array}{l}\text { Married/ } \\
\text { cohabitating } \\
\text { with children } \\
\text { at home }\end{array}$ & $260(8.17)$ & 465 (14.6) & $159(4.99)$ & 436 (13.69) & & & \\
\hline
\end{tabular}


Table 4 (continued)

\begin{tabular}{|c|c|c|c|c|c|c|c|}
\hline & $\begin{array}{l}\text { Trajectory 1: } \\
\text { low direct and } \\
\text { indirect costs }\end{array}$ & $\begin{array}{l}\text { Trajectory 2: } \\
\text { high direct but } \\
\text { low indirect } \\
\text { costs }\end{array}$ & $\begin{array}{l}\text { Trajectory } 3: \text { low } \\
\text { direct but high } \\
\text { indirect costs }\end{array}$ & $\begin{array}{l}\text { Trajectory 4: } \\
\text { high direct and } \\
\text { indirect costs }\end{array}$ & $\begin{array}{l}\text { Pearson's Chi } \\
\text { square ( } p \text { value) }\end{array}$ & $\begin{array}{l}\text { Log-likelihood } \\
\text { test Chi square } \\
\text { ( } p \text { value) }\end{array}$ & Diff. in $\mathrm{R} 2^{\mathrm{c}}$ \\
\hline & $19.9 \%$ & $31.8 \%$ & $16.1 \%$ & $32.1 \%$ & & & \\
\hline & $N(\%)^{\mathrm{a}}$ & $N(\%)^{\mathrm{a}}$ & $N(\%)^{\mathrm{a}}$ & $N(\%)^{\mathrm{a}}$ & & & \\
\hline $\begin{array}{l}\text { Single without } \\
\text { children at } \\
\text { home }\end{array}$ & $236(7.41)$ & $395(12.41)$ & $176(5.53)$ & 359 (11.28) & & & \\
\hline $\begin{array}{l}\text { Single with } \\
\text { children at } \\
\text { home }\end{array}$ & $61(1.92)$ & $51(1.6)$ & $65(2.04)$ & $126(3.96)$ & & & \\
\hline Multi-morbidity & & & & & $\begin{array}{l}191.00 \\
\quad(<0.0001)\end{array}$ & $\begin{array}{l}190.52 \\
\quad(<0.0001)\end{array}$ & 0.029 \\
\hline Yes & $1284(39.24)$ & $187(5.72)$ & $181(5.53)$ & $1375(42.02)$ & & & \\
\hline No & $136(4.16)$ & $58(1.77)$ & $31(0.95)$ & $20(0.61)$ & & & \\
\hline $\begin{array}{l}\text { Anxiety/depres- } \\
\text { sion }\end{array}$ & & & & & $5.64(0.131)$ & $5.57(0.135)$ & 0.007 \\
\hline Yes & 55 (1.68) & $50(1.53)$ & $40(1.22)$ & $36(1.1)$ & & & \\
\hline No & $738(22.56)$ & $801(24.48)$ & $781(23.87)$ & $771(23.56)$ & & & \\
\hline
\end{tabular}

${ }^{a}$ The percentages are calculated as $n$ divided with the total number of individuals in the study population (3272 if not otherwise indicated; see ${ }^{\mathrm{b}}$ )

${ }^{\mathrm{b}}$ The total number of individuals with this type of information in the study population (i.e. all individuals excluding those with missing information in this category) was 3184

${ }^{\mathrm{c}}$ The $\mathrm{R} 2$ for the full model was 0.178

Additional analysis was performed to see whether the main cost-of-illness findings would still be applicable for where MS was mentioned as the main cause/diagnosis. Overall, approximately half of direct costs and even less for indirect costs were "due to MS", i.e. MS was reported as the main diagnosis. Still, this does not imply that the rest of the healthcare resources that SA and DP consumed were not related to MS symptoms/progression. Many other reasons for healthcare or SA/DP can be related to the MS diagnosis, e.g. mental disorders [27]. Also, some people might already have been on long-term SA or DP before an MS diagnosis was established, having a different diagnosis for the SA/DP spell than MS. In this case, the other diagnosis, and not MS, would be the main diagnosis of the SA/DP spell, even after MS diagnosis was established [28].

\subsection{Strengths and Limitations}

The main strengths of this study are its prospective cohort design and that data from administrative registers could be used, instead of self-reported information. All people diagnosed with MS between 2006 and 2009 could be followed for several years in nationwide registers of high quality [29, 30], eliminating the selection biases that some previous MS studies had due to recruitment of only a sub-set of people with MS [4]. For this study, it would be useful to have more years of follow-up, in order to identify any further increase or decrease of the cost of illness of people with MS over a longer period of time.

It is also an advantage that a societal perspective could be applied in this study, i.e. indirect costs were also included. High-quality information from the MiDAS register in Sweden, capturing with accuracy the number of SA and DP days per year, allowed for detailed calculations of the productivity losses of MS patients, which are considered the main longterm driver of the cost of illness of MS. However, a limitation of this study is the fact that short-term SA spells of 14 days or fewer were not quantified into indirect costs. Therefore, it is possible that we have underestimated indirect costs. Moreover, any productivity reductions while being present at work that could potentially be related to the presence of MS were not quantified. Furthermore, productivity losses incurred by partners of people with MS, i.e. informal care costs, were not included in the cost-of-illness calculations; while measuring these costs was beyond the aim of this study, they are an important cost component when defining the overall cost of illness of MS [4, 7, 8, 22]. In addition, the use of other macroeconomic indicators for defining indirect costs, e.g. gross value added, would augment the interpretation of our findings.

Another important limitation is the absence of information regarding the clinical characteristics (e.g. disease phenotype) and disability level of people with MS. Both direct and indirect costs have been shown to be strongly correlated with disability levels in previous studies [4, 7, 8, 22]. Therefore, the 
differences between the observed cost-of-illness trajectories found in our study might have been further explained if such information had been available. On the other hand, it is not certain that the association of the sociodemographic characteristics and multi-morbidity with the observed trajectories would be altered had such clinical information been available. There is lack of knowledge regarding the association of sociodemographic characteristics with the clinical characteristics (e.g. disability and phenotype) of MS.

Moreover, it would have been an advantage if cost information on hospital-administered DMTs (i.e. natalizumab, intravenous immunoglobulin, mitoxantrone, and rituximab), primary healthcare, rehabilitation, home help, and home investments to improve mobility was available to include in our cost-of-illness calculations. Since our focus was on newly diagnosed people with MS, when the expected main cost driver for direct costs is drugs, the absence of DMT cost information for hospital-administered drugs would play a role. In addition, information for primary healthcare visits and rehabilitation would provide a better picture regarding the cost of illness of MS patients in Sweden. These other cost components usually become more prominent with disease progression, which would have affected our trajectory analysis significantly had we had a longer follow-up.

\section{Conclusions}

In this study, register data with nationwide coverage were used to explore the heterogeneity in the cost-of-illness development over time among newly diagnosed people with MS. Not everyone had a high cost of illness of MS immediately after diagnosis, a fact that can be possibly linked to the underlying disease severity and/or treatment with DMTs, but also to socioeconomic characteristics and multi-morbidity. This information can be used when assessing the development of the cost of illness of MS in relation to disease progression, and for understanding the impact that innovative MS therapies, which can slow disease progression, have on the cost of illness of the disease.

Author Contributions All authors (Korinna Karampampa, KK; Hanna Gyllensten, HG; Fei Yang, FY; Chantelle Murley, CM; Emilie Friberg, EF; Jan Hillert, JH; and Kristina Alexanderson, KA) contributed to the conceptualisation of the research questions, the study design and methods. KK performed the analysis of the data, interpreted the study findings, and drafted the manuscript. All remaining authors (HG, FY, $\mathrm{CM}, \mathrm{EF}, \mathrm{JH}$, and $\mathrm{KA}$ ) assisted in the interpretation of the study findings and contributed to the manuscript with comments/suggestions and text.

Data Availability Statement The data used in this study is administered by the Division of Insurance Medicine, Karolinska Institutet, and cannot be made public. According to the General Data Protection Regulation, Swedish law SFS 2018:218, the Swedish Data Protection Act, the Swedish Ethical Review Act, and the Public Access to Information and
Secrecy Act, this type of sensitive data can only be made available, after legal review, to researchers who meet the criteria for access to this type of sensitive and confidential data. Readers may contact Professor Kristina Alexanderson (kristina.alexanderson@ki.se) regarding the data.

\section{Compliance with Ethical Standards}

Funding All authors (KK, HG, FY, CM, EF, JH, and KA) have received funding from an unrestricted investigator-initiated trial grant from Biogen to support the conduct of this study.

Conflict of interest All authors (KK, HG, FY, CM, EF, JH, and KA) are employed at or affiliated with the Department of Clinical Neuroscience, Karolinska Institutet, Stockholm, Sweden. FY is currently employed by PPD Scandinavia AB, and HG is currently employed part-time by Statfinn/EPID Research (which is part of IQVIA); both companies are contract research organisations that perform commissioned pharmacoepidemiological studies and, therefore, are collaborating with several pharmaceutical companies.

Open Access This article is distributed under the terms of the Creative Commons Attribution-NonCommercial 4.0 International License (http://creativecommons.org/licenses/by-nc/4.0/), which permits any noncommercial use, distribution, and reproduction in any medium, provided you give appropriate credit to the original author(s) and the source, provide a link to the Creative Commons license, and indicate if changes were made.

\section{References}

1. Jo C. Cost-of-illness studies: concepts, scopes, and methods. Clin Mol Hepatol. 2014;20:327-37.

2. Ahlgren C, Oden A, Lycke J. High nationwide incidence of multiple sclerosis in Sweden. PLoS One. 2014;9:e108599.

3. Gyllensten H, Wiberg M, Alexanderson K, et al. Costs of illness of multiple sclerosis in Sweden: a population-based register study of people of working age. Eur J Health Econ. 2017;19:435-46.

4. Ernstsson O, Gyllensten H, Alexanderson K, et al. Cost of illness of multiple sclerosis-a systematic review. PLoS One. 2016;11:e0159129.

5. Gyllensten H, Kavaliunas A, Alexanderson K, et al. Costs and quality of life by disability among people with multiple sclerosis: a register-based study in Sweden. Mult Scler J Exp Transl Clin. 2018;4:2055217318783352.

6. Brundin L, Kobelt G, Berg J, et al. New insights into the burden and costs of multiple sclerosis in Europe: results for Sweden. Mult Scler. 2017;23:179-91.

7. Karampampa K, Gustavsson A, Miltenburger C, et al. Treatment experience, burden and unmet needs (TRIBUNE) in MS study: results from five European countries. Mult Scler. 2012;18:7-15.

8. Kobelt G, Berg J, Lindgren P, et al. Costs and quality of life of patients with multiple sclerosis in Europe. J Neurol Neurosurg Psychiatry. 2006;77:918-26.

9. Gyllensten H, Kavaliunas A, Murley C, et al. Costs of illness progression for different multiple sclerosis phenotypes: a populationbased study in Sweden. Submitted. 2018.

10. Allebeck P, Mastekaasa A. Swedish Council on Technology Assessment in Health Care (SBU). Chapter 5. Risk factors for sick leave-general studies. Scand J Public Health Suppl. 2004;63:49-108. 
11. World Health Organization. International statistical classification of diseases and related health problems 10th revision (ICD-10). Accessed on 12 Dec 2018. https://icd.who.int/browse10/2016/en.

12. Ludvigsson JF, Almqvist C, Bonamy AK, et al. Registers of the Swedish total population and their use in medical research. Eur J Epidemiol. 2016;31:125-36.

13. Drummond MF, Sculpher MJ, Claxton K, et al. Methods for the economic evaluation of health care programmes. Oxford: Oxford University Press; 2015.

14. National Insurance Agency [Försäkringskassan]-Social insurance in figures 2017. https://www.forsakringskassan.se/wps/wcm/ connect/6fa0e434-a212-4e6b-8c8d-5d7a498a253d/socialforsakrin gen-siffror-2017-engelsk.pdf?MOD=AJPERES\&CVID. Accessed 12 Dec 2018.

15. Eurostat. HICP - all items - annual average indices $(2015=100)$. Accessed on December 12, 2018. https://ec.europa.eu/eurostat/ $\operatorname{tgm} /$ table. do $?$ tab $=$ table \&init $=1 \&$ language $=$ en $\&$ pcode $=$ tec 00 $027 \&$ plugin $=1$.

16. Kim TK. T test as a parametric statistic. Korean J Anesthesiol. 2015;68:540-6.

17. Nagin DS, Jones BL, Passos VL, et al. Group-based multi-trajectory modeling. Stat Methods Med Res. 2018;27:2015-23.

18. Jones BL, Nagin DS, Roeder K. A SAS procedure based on mixture models for estimating developmental trajectories. Sociol Methods Res. 2001;29:374-93.

19. Agresti A. Categorical data analysis. New York: John Wiley \& Sons; 2003.

20. Pratt NL, Kerr M, Barratt JD, et al. The validity of the Rx-risk comorbidity index using medicines mapped to the anatomical therapeutic chemical (ATC) classification system. BMJ Open. 2018;8:e021122.

21. O'Connell K, Kelly SB, Fogarty E, et al. Economic costs associated with an MS relapse. Mult Scler Relat Disord. 2014;3:678-83.

22. Kobelt G, Thompson A, Berg J, et al. New insights into the burden and costs of multiple sclerosis in Europe. Mult Scler. 2017;23:1123-36.

23. Schwenkenbecher P, Sarikidi A, Wurster U, et al. McDonald criteria 2010 and 2005 compared: persistence of high oligoclonal band prevalence despite almost doubled diagnostic sensitivity. Int J Mol Sci. 2016;17:1592.

24. Gyllensten H, Wiberg M, Alexanderson K, et al. Costs of illness of multiple sclerosis in Sweden: a population-based register study of people of working age. Eur J Health Econ. 2018;19:435-46.

25. Rieckmann P, Centonze D, Elovaara I, et al. Unmet needs, burden of treatment, and patient engagement in multiple sclerosis: a combined perspective from the MS in the 21 st century steering group. Mult Scler Relat Disord. 2018;19:153-60.

26. Kavaliunas A, Manouchehrinia A, Stawiarz L, et al. Importance of early treatment initiation in the clinical course of multiple sclerosis. Mult Scler. 2017;23:1233-40.
27. Brenner P, Alexanderson K, Bjorkenstam C, et al. Psychiatric diagnoses, medication and risk for disability pension in multiple sclerosis patients; a population-based register study. PLoS One. 2014;9:e104165.

28. Gyllensten H, Wiberg M, Alexanderson K, et al. How does work disability of patients with MS develop before and after diagnosis? A nationwide cohort study with a reference group. BMJ Open. 2016;6:e12731.

29. Ludvigsson JF, Andersson E, Ekbom A, et al. External review and validation of the Swedish national inpatient register. BMC Public Health. 2011;11:450.

30. Wallerstedt SM, Wettermark B, Hoffmann M. The first decade with the Swedish Prescribed Drug Register-a systematic review of the output in the scientific literature. Basic Clin Pharmacol Toxicol. 2016;119:464-9.

31. Swedish Association of Local Authorities and Regions [Sveriges Kommuner och Landsting]—KPP Somatik. https://skl.se/ekono mijuridikstatistik/statistik/kostnadperpatientkpp/kppsomatik .1077.html. Accessed 12 Dec 2018.

32. 1177 Vårdguiden - Patient fees in Västra Götaland county in Sweden [Patientavgifter i Västra Götaland]. https://www.1177.se/Vastr a-Gotaland/Regler-och-rattigheter/Patientavgifter-i-Vastra-Gotal and/. Accessed 12 Dec 2018.

33. Swedish Association of Local Authorities and Regions [Sveriges Kommuner och Landsting]—Patientavgifter fr.o.m. den 1 januari 2018. https://skl.se/download/18.3ee4cc4f1611af77b891 79dd/1517495154691/avgift_\%C3\%B6ppenv\%C3\%A5rd_ink1. missiv_slutenavg18_19jan18.pdf. Accessed 12 Dec 2018.

34. Swedish Association of Local Authorities and Regions [Sveriges Komuner och Landsting]-Patientavgifter i hälso- och sjukvården 2018. https://skl.se/halsasjukvard/patientinflytande/patientavgifter .14668.html. Accessed 12 Dec 2018.

35. Statistics Sweden. Genomsnittlig månadslön, lön i fasta priser och lönespridning efter yrkesgrupp (SSYK 96) och kön. År 1996-2013-Statistikdatabasen. Accessed on 12 Dec 2018. http:// www.statistikdatabasen.scb.se/pxweb/sv/ssd/START_AM AM0112/TidsserieYrke/.

36. Swedish Tax Authority [Skatteverket]. Belopp och procent för åren 2014-2004-privat. Accessed on 12 Decr 2018. https://www. skatteverket.se/privat/skatter/beloppochprocent/tidigarear.4.1a098 b721295c544e1f800018171.html.

37. Eurostat. Annual exchange rates (ECU/EUR exchange rates versus national currencies). Accessed on 12 December 2018. https ://ec.europa.eu/eurostat/tgm/table.do?tab=table\&init=1\&langu age $=$ en $\&$ pcode $=$ tec $00033 \&$ plugin $=1$. 